\title{
Les jachères de légumineuses arborescentes: sources potentielles de bois énergie et de service en Côte d'Ivoire
}

\author{
GNAHOUA Guy Modeste ${ }^{1}$, NGUESSAN Kanga' ${ }^{1}$ Anatole, BALLÉ Pity ${ }^{2}$ \\ 1. Centre National de Recherche Agronomique 08 B.P. 801 Abidjan 08, Côte d'Ivoire \\ 2. Centre de Recherche en Écologie 08 B.P. 109 Abidjan 08, Côte d'Ivoire \\ Corresponding author : gm.gnahoua@hotmail.fr
}

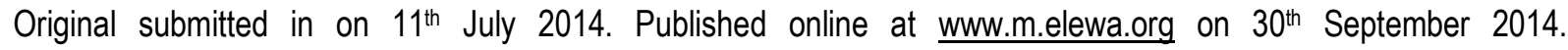
http://dx.doi.org/10.4314/jab.v81i1.11

\section{RESUME}

Objectifs : Ce travail étudie les aptitudes des jachères de légumineuses arborescentes à produire du bois d'utilisation courante pour pallier les pénuries de ligneux. Plus spécifiquement, la présente étude vise à montrer les quantités de bois que peuvent produire les jachères de Légumineuses arborescentes, et les formes d'utilisations de ce bois.

Méthodologie et Résultats: Les arbres de quatre jachères améliorées ont été mesurés (hauteur totale et diamètre à $1,30 \mathrm{~m}$ ) avant l'abattage. Le poids sec du bois produit a été déterminé et les troncs d'arbres testés en bois de chauffe, charbon et perches. Les résultats ont montré des hauteurs allant de 9,45 m chez Lebbeck (Albizia lebbeck) à 17,66 m chez Mangium (Acacia mangium), en seulement 6 ans. La production de bois la plus importante (138 t.ha-1 chez Acacia mangium), représentait une productivité de 23 t.ha-1an-1 $^{-1}$. Concernant les utilisations possibles, Acacia mangium et Leucaena leucocephala ont produit les meilleures perches tandis qu'Acacia auriculiformis et Albizia lebbeck ont fourni du bois énergie et du charbon de bonne qualité.

Conclusion et application : La plantation d'une jachère de légumineuses arborescentes permet (outre l'effet des arbres sur la fertilité des sols) de produire, en peu de temps, une quantité suffisante de bois d'utilisation courante. La production de perches, de poteaux et la transformation en charbon d'une partie du bois sont des potentialités intéressantes de ces jachères de Légumineuses. Leur commercialisation procure des ressources additionnelles et font de ces nouveaux types de jachères un moyen de diversification des revenus en milieu rural. Ainsi, les jachères de légumineuses arborescentes sont à promouvoir comme pratiques alternatives à la jachère naturelle pour permettre la production de ligneux et contribuer à freiner la déforestation des terroirs ruraux.

Mots clés : agroforesterie, jachères améliorées, légumineuses arborescentes, production de bois.

\section{ABSTRACT}

Fallow Leguminous trees: potential sources of fuel wood and services in Côte d'Ivoire

Objective: This study aims at monitoring the potentials of fallow leguminous trees to produce multipurpose wood in order to address wood shortage. Specifically, the current study aims to quantify the production of wood and assess their possible uses in rural area. 

bois énergie et de service en Cote d'Ivoire

Methodology and Results: Trees in four improved fallows were measured (height and diameter) before felling. Wood produced by trees was weighed and assessed as fire wood, charcoal and poles through a survey. Results showed tree heights ranging from 9,45 $\mathrm{m}$ (Albizia lebbeck) to 17,66 $\mathrm{m}$ (Acacia mangium) within only 6 years. Wood production reached 138 t.ha-1 $^{-1}$ in Acacia mangium plots, meaning a productivity of 23 tons.ha${ }^{1}$ year-1. ${ }^{-}$. Regarding the possible uses of fallow products, Acacia mangium and Leucaena leucocephala produced the best poles whilst Acacia auriculiformis and Albizia lebbeck provided good quality fire wood and charcoal. Conclusion and application: This study suggests that planting leguminous tree fallow permits the production of a high amount of multipurpose wood, within a short term, in addition to tree effects on soil fertility. The potentials of these types of fallows are poles, firewood and charcoal production which can provide financial resources and make leguminous tree plantation a means of income diversification in rural area. Consequently, fallow leguminous tree are to be promoted as alternative practices to natural short term fallowing in order to produce wood and contribute to slow forest degradation.

Keywords: agroforestry, improved fallow, leguminous trees, wood production.

\section{INTRODUCTION}

Le bois constitue encore la principale source d'énergie des ménages africains (Ouédraogo, 2007). En Afrique de l'Ouest, les besoins annuels de la ville de Niamey étaient estimés à 150.000 tonnes de bois au cours de la décennie 1990-2000 (Montagne et Housseini, 2000). Selon Bisiaux et al. (2009), les besoins annuels en charbon de bois de la mégapole de Kinshasa (8 millions d'habitants) sont de 0,6 à 1,2 millions de tonnes. En Afrique, le bois d'utilisation courante provient du défrichement des formations forestières et des prélèvements effectués dans les jachères en reconstitution. Cependant, en Côte d'Ivoire, la pression de l'agriculture a exacerbé la déforestation des zones rurales et réduit la durée des jachères naturelles qui permettait de restaurer la fertilité des sols et de régénérer les ressources ligneuses (Floret et Pontanier, 2000). Les jachères naturelles actuelles, de courtes durées ( 3 à 5 ans), sont envahies par une herbacée, l'Eupatorium; Chromolaena odorata (Asteraceae) qui inhibe la régénération naturelle des espèces ligneuses (de Rouw, 1993), d'où la raréfaction actuelle de bois dans les campagnes. Pour mettre au point des itinéraires agricoles alternatifs permettant de produire du bois d'utilisation courante, les jachères plantées en légumineuses arborescentes ont fait l'objet de nombreuses études (Harmand, 1988, Peltier et Eyog-Matig, 1989 ; Peltier et al., 1995). Les légumineuses se subdivisent en trois grandes sous familles: Caesalpiniaceae, Mimosaceae et Papillionaceae). La plupart des légumineuses fixent l'azote atmosphérique en symbiose avec des bactéries du genre Rhizobium, ce qui leur confère une relative autonomie de nutrition azotée ainsi qu'une grande aptitude à se développer sur des sols pauvres en éléments nutritifs. En Afrique, certaines espèces de légumineuses sont caractéristiques des agroécosystèmes et des zones habitées. On peut citer le Dabema (Piptadeniastrum africanum) qui produit un excellent bois d'œuvre, le flamboyant (Delonix regia), une plante ornementale et le soja (Glycine max) qui est une espèce alimentaire et fourragère. Les légumineuses arborescentes sont dotées de rythmes de croissance très rapides (Dommergues et al., 1999 ; Singh et al., 2004). Elles ont un fort potentiel de production de litière (Bernhard-Reversat et al., 1993 ; Gnahoua et al., 2013) et de bois (Dupuy et Nguessan, 1990). Sein et Mitlöhner (2011) estiment à 190,3 tha-1 la biomasse aérienne (bois et feuilles) de jeunes peuplements d'Acacia mangium de 5 à 7 ans, au Vietnam. En Asie tropicale, outre le bois d'énergie, les produits issus des plantations de légumineuses arborescentes font l'objet de nombreux usages : bois d'œuvre et d'ébénisterie, poteaux de construction, perches, artisanat, fourrage, etc. (Krisnawati et al., 2011). Dans la perspective de pallier les pénuries actuelles de bois d'utilisation courante par la plantation de jachères de légumineuses arborescentes, en milieu rural de Côte d'Ivoire, la présente étude a pour objet d'évaluer la production et l'utilisation du bois de quatre peuplements d'arbres fixateurs d'azote. 
MATERIEL ET METHODES

Site de l'étude : L'étude s'est déroulée à la Station de recherche forestière du CNRA à Oumé $\left(06^{\circ} 17 \mathrm{LN}\right.$ et $05^{\circ} 31$ LO) située en région Centre Ouest de Côte d'Ivoire. Le climat est subéquatorial avec deux saisons de pluies et deux saisons sèches. La température moyenne annuelle est de $26,5^{\circ} \mathrm{C}$. La pluviométrie observée au cours des 20 dernières années se situe en moyenne à $1350 \mathrm{~mm}$ par an. Les sols sont ferralitiques moyennement désaturés et globalement bien drainés. La végétation d'origine est de type forêt dense humide semi décidue (Guillaumet et Adjanohoun, 1971).

Matériel végétal : Le matériel végétal est constitué de quatre espèces de légumineuses arborescentes (Mimosacées) : Acacia mangium Willd., Acacia auriculiformis A. Cunn., Albizia lebbeck (L) Benth. et Leucaena leucocephala Willd.

Dispositif expérimental L'essai est un dispositif d'enrichissement de jachères âgé de 6 ans, comportant 6 blocs et 5 traitements. Les traitements correspondent aux 4 espèces de Légumineuses conduites en peuplement d'amélioration de jachères (Acacia auriculiformis, Acacia mangium, Albizia lebbeck et Leucaena leucocephala) età la jachère naturelle (témoin) dominée par Chromolaena odorata. Les légumineuses ont été plantées à l'écartement de $2 \mathrm{~m} \times 2 \mathrm{~m}$ (soit 2500 tiges.ha-1). Chaque parcelle unitaire mesure $10 \mathrm{~m} \times 10 \mathrm{~m}\left(100 \mathrm{~m}^{2}\right)$ et comporte 36 arbres. La présente étude ayant porté uniquement sur les produits ligneux, la jachère témoin de Chromolaena odorata (herbacée) n'a pas été prise en compte.

Diamètre et hauteur des peuplements de légumineuses : Avant l'abattage des peuplements, leurs caractéristiques dendrométriques ont été déterminées; la hauteur totale a été mesurée à l'aide d'un instrument de mesure optique, le "Blum-leiss » (Parde et Bouchon, 1988). Le diamètre a été déduit de la circonférence

\section{RESULTATS}

Croissance des peuplements : hauteur et diamètre : A six (6) ans, la majorité des espèces étudiées ont dépassé 9 mètres de haut (Tableau 1). La hauteur moyenne la plus importante était celle d'Acacia mangium $(17,66 \mathrm{~m})$ et celle la plus petite a été observée chez Albizia lebbeck $(9,45 \mathrm{~m})$. Les accroissements moyens correspondant étaient de 3,08 m.an-1 pour l'Acacia et de 1,7 m.an-1 pour mesurée au ruban métrique, à 1,30 mètre du sol. La circonférence permet de faire une stratitification des arbres d'un peuplement selon leur grosseur: gros, moyens et petits. La connaissance de la hauteur et du diamètre permet de déterminer les différents accroissements annuels d'un peuplement.

Estimation de la biomasse ligneuse : Pour déterminer la biomasse ligneuse, un échantillon de 26 à 35 arbres par espèce a été abattu, sur la base de leurs circonférences (gros arbres, moyens et petits). Les arbres abattus ont été découpés à la tronçonneuse et séparés en deux compartiments de biomasse (bois fort, de diamètre $>3 \mathrm{~cm}$ et petits bois de diamètre $<3 \mathrm{~cm}$ ). Les billons ont été pesés avec une bascule à $100 \mathrm{~g}$ de précision. Pour chaque espèce, un échantillon composite de bois fort et de petits bois a été prélevé et séché à l'étuve à $80^{\circ} \mathrm{C}$ jusqu'à poids constant. La biomasse ligneuse de chaque espèce (en t.ha-1) a été déterminée à partir des poids secs des échantillons étuvés. Le volume de bois produit (en $\mathrm{m}^{3} \mathrm{ha}^{-1}$ ) a été déterminé en utilisant la densité (ou masse volumique) du bois de chaque espèce. Usages potentiels du bois des jachères améliorées : Le bois produit par les Légumineuses a été testé aux diverses utilisations du milieu rural, à savoir : perches pour la construction, bois de chauffe et charbon de bois. Vingt ménages des villages riverains de la Station de Recherche ont été interrogés sur la qualité et les formes d'utilisation possibles du bois produit par les Légumineuses étudiées. Pour la production de charbon, 6 à 8 stères de bois de chaque espèce ont été carbonisés dans des meules traditionnelles en terre.

Analyses des données: Les données des différentes mesures et déterminations ont fait l'objet d'analyses de variance (Dagnelie, 1973) à l'aide du logiciel XLSTAT aux seuils de probabilité de $5 \%$ et $1 \%$.

Albizia lebbeck. Concernant le diamètre, ces mêmes espèces, Acacia mangium et Albizia lebbeck, ont affiché respectivement la valeur la plus élevée $(14,33 \mathrm{~cm})$ et celle la plus faible $(7,96 \mathrm{~cm})$, ce qui correspond à des accroissements moyens annuels de 2,4 et $1,33 \mathrm{~cm}$ respectivement. 
Gnahoua et al. J. Appl. Biosci. 2014. Les jachères de légumineuses arborescentes: Sources potentielles de bois énergie et de service en Cote d'Ivoire

Tableau 1: Critères de vigueur des légumineuses arborées étudiées, à 6 ans.

\begin{tabular}{lcccc}
\hline Espèce & $\begin{array}{c}\text { Acacia } \\
\text { mangium }\end{array}$ & $\begin{array}{c}\text { Acacia } \\
\text { auriculiformis }\end{array}$ & $\begin{array}{c}\text { Albizia } \\
\text { lebbeck }\end{array}$ & $\begin{array}{c}\text { Leucaena } \\
\text { leucocephala }\end{array}$ \\
\hline Hauteur $(\mathrm{m})$ & $17,66 \mathrm{a}$ & $14,16 \mathrm{~b}$ & $9,45 \mathrm{c}$ & $14,53 \mathrm{~b}$ \\
Diamètre $(\mathrm{cm})$ & $14,94 \mathrm{a}$ & $14,26 \mathrm{a}$ & $8,14 \mathrm{~b}$ & $8,52 \mathrm{~b}$ \\
Accroissement en hauteur $(\mathrm{m} / \mathrm{an})$ & 3,08 & 2,2 & 1,7 & 2,42 \\
Accroissement en diamètre $(\mathrm{cm} / \mathrm{an})$ & 2,4 & 1,64 & 1,33 & 1,4 \\
\hline
\end{tabular}

Deux moyennes affectées de la même lettre ne diffèrent pas par le test de Newman-Keuls à $P=0,05$

Production de bois par les jachères : Les productions de bois d'Acacia mangium (138 t.ha-1) et d'A.auriculiformis (134 t.ha-1) ont été les plus importantes tandis qu'Albizia lebbeck (68 tha- $\left.{ }^{-1}\right)$ a affiché les plus petites quantités. En termes de productivité, les espèces étudiées ont montré une accumulation de biomasse de 11,33 à 23 t.ha- $^{-1}$. an-1. . La figure 1 montre une pile de bois d'Acacia mangium issu de l'abattage des peuplements étudiés et destiné à la carbonisation.

Tableau 2 : Production de bois, des peuplements de légumineuses arborées âgés de 6 ans.

\begin{tabular}{lcccc}
\hline Espèce & $\begin{array}{c}\text { Acacia } \\
\text { mangium }\end{array}$ & $\begin{array}{c}\text { Acacia } \\
\text { auriculiformis }\end{array}$ & $\begin{array}{c}\text { Albizia } \\
\text { lebbeck }\end{array}$ & $\begin{array}{c}\text { Leucaena } \\
\text { leucocephala }\end{array}$ \\
\hline Production de bois (t.ha-1) & $138 \mathrm{a}$ & $134 \mathrm{a}$ & $68 \mathrm{~b}$ & $114 \mathrm{a}$ \\
Productivité $\left(\right.$ t.ha- $\left.^{-1} \cdot \mathrm{an}^{-1}\right)$ & 23 & 22,33 & 11,33 & 19
\end{tabular}

Deux moyennes affectées de la même lettre ne diffèrent pas par le test de Newman-Keuls à $P=0,05$

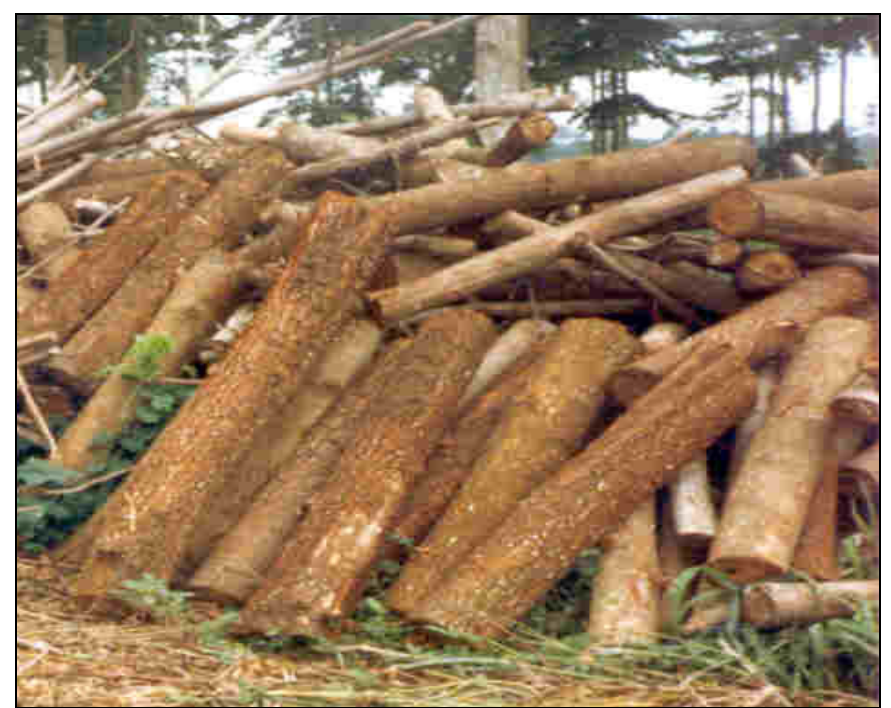

Figure 1 : Piles de bois fort et de branchages d'Acacia mangium avant la carbonisation

Formes d'utilisation des produits ligneux issus des jachères améliorées : Pour les trois formes d'utilisation possible du bois des Légumineuses étudiées, les aptitudes décrites par les paysans enquêtés sont présentées au tableau 3. A part Acacia mangium dont la combustion a produit une grande quantité de cendres, les trois autres espèces ont présenté de bonnes qualités de bois de chauffe. Acacia mangium et Leuceana leucocephala qui ont des qualités voisines (hautes tiges et ports droits) ont produit les meilleures perches tandis que Acacia auriculiformis et Albizia lebbeck (aux bois plus denses) se sont montrées plus adaptées à la carbonisation. Globalement, les espèces en présence ont montré des qualités moyennes à bonnes pour les différentes formes d'utilisation de la ressource bois en milieu rural. 
Gnahoua et al. J. Appl. Biosci. 2014. Les jachères de légumineuses arborescentes: Sources potentielles de bois énergie et de service en Cote d'Ivoire

Tableau 3 : Aptitudes du bois des jachères étudiées aux principales utilisations du milieu rural

\begin{tabular}{lcccc}
\hline Espèce & $\begin{array}{c}\text { Acacia } \\
\text { auriculiformis }\end{array}$ & $\begin{array}{c}\text { Acacia } \\
\text { mangium }\end{array}$ & $\begin{array}{c}\text { Albizia } \\
\text { lebbeck }\end{array}$ & $\begin{array}{c}\text { Leucaena } \\
\text { leucocephala }\end{array}$ \\
\hline Carbonisation & bonne & moyenne & bonne & moyenne \\
Perches & moyenne & bonne & mauvaise & bonne \\
Chauffage & bonne & mauvaise & bonne & bonne \\
\hline
\end{tabular}

Carbonisation du bois et revenus financiers : L'essai de carbonisation réalisé dans des meules traditionnelles en terre (figure 2) a donné des rendements de $18 \%$ (Acacia auriculiformis) et de 21\% (Albizia lebbeck). Ces deux espèces ont produit du charbon de bonne qualité et nous ont permis d'estimer les recettes que la carbonisation des essences étudiées peut générer (tableau 4). Ainsi, avec Acacia auriculiformis et Albizia lebbeck, la production de charbon est respectivement de 24,12 t.ha-1 ${ }^{-1}$, soient 344 sacs de $70 \mathrm{~kg}$ et de 14,28 t.ha-1, $^{-1}$ ce qui représente 204 sacs de $70 \mathrm{~kg}$. Ce charbon vendu bord champ au prix de $5000 \mathrm{~F}$ le sac de $70 \mathrm{~kg}$ procure au planteur de jachères arborées des recettes de 1.020 .000 à $1.720 .000 \mathrm{~F}$ à l'hectare. Cependant, il convient de noter que les coûts de production de ce charbon n'ont pu être déterminés.

Tableau 4 : Production de charbon et recettes estimées de l'exploitation de la jachère arborée

\begin{tabular}{lllll}
\hline Espèce & $\begin{array}{l}\text { Production } \\
\text { de bois (t.ha-1) }\end{array}$ & $\begin{array}{l}\text { Rendement } \\
\text { en charbon } \\
\text { (t.ha-1) }\end{array}$ & $\begin{array}{l}\text { Nombre de } \\
\text { sacs de } \\
\mathbf{7 0 ~ k g}\end{array}$ & $\begin{array}{l}\text { Coût bord champ } \\
\text { (5000 F. le sac) }\end{array}$ \\
\hline A. auriculiformis & 134 & 24,12 & 344 & $1.720 .000 \mathrm{~F}$ \\
Albizia. lebbeck & 68 & 14,28 & 204 & $1.020 .000 \mathrm{~F}$ \\
\hline
\end{tabular}

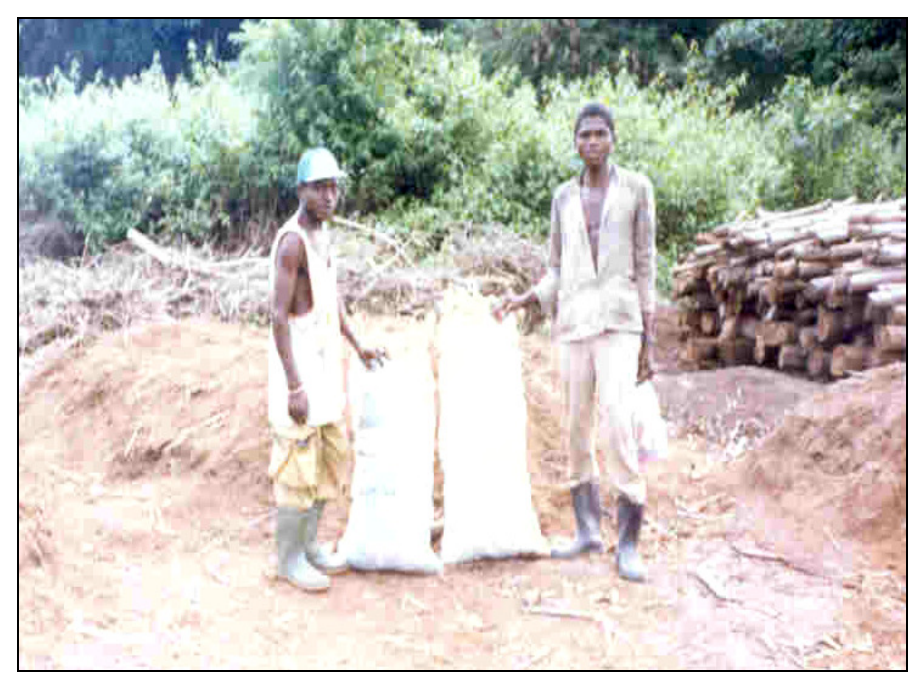

Figure 2 : Production de charbon en meules traditionnelles (pile de bois de Légumineuses et sacs de charbon)

\section{DISCUSSION}

Hauteur et circonférence : A cinq ans, la hauteur des espèces étudiées a oscillé entre 9,45 et $17,67 \mathrm{~m}$ et les diamètres entre 8,14 et $14,94 \mathrm{~cm}$. Ces valeurs dénotent de la croissance rapide des essences en présence et sont identiques à celles de Dupuy et N'guessan (1991) qui ont observé, en basse Côte d'lvoire, des pieds d'Acacia mangium de 15 à 18,3 m de hauteur, à cinq ans, avec des diamètres de 12 à $13,7 \mathrm{~cm}$. Les mêmes auteurs rapportent qu'Acacia auriculiformis a atteint $13 \mathrm{~m}$ de haut, en cinq (5) ans avec un diamètre moyen de $12 \mathrm{~cm}$. Chez Acacia auriculiformis de 4 ans et 4 mois planté sur le littoral ivoirien, Lekadou et al. (2009) ont observé des hauteurs de 7,2 à $8,8 \mathrm{~m}$ et des diamètres compris entre 10,3 et 12, $10 \mathrm{~cm}$. En Inde, Albizia lebbeck « Indian siris » est connue pour sa croissance rapide : $18 \mathrm{~m}$ de hauteur et $66 \mathrm{~cm}$ de diamètre en 10 ans, soit des 

bois énergie et de service en Cote d'Ivoire

accroissements moyens annuels respectifs de $1,8 \mathrm{~m}$ et $6,6 \mathrm{~cm}$ (Prinsen, 1986). Leucaena leucocephala a montré aux Philippines, un rythme annuel de croissance estiméà $2,5 \mathrm{~m}$ pour la hauteur et $5 \mathrm{~cm}$ sur le diamètre selon NAS (1984) et atteint $20 \mathrm{~m}$ de haut en 8 ans. Globalement, les critères de vigueur enregistrés sur les mêmes légumineuses dans des conditions écologiques similaires sont cohérents avec celles de la présente étude.

Production de bois : La production de bois de notre étude (134 et 138 t.ha $^{-1}$ en 6 ans) chez Acacia mangium et Acacia auriculiformis a été comparée à celle d'études similaires et s'est montrée plus élevée. Dans des peuplements d'Acacias australiens plus âgés ( 8 ans), au Sud de la Côte d'Ivoire, Dupuy et Nguessan (1991) ont noté des productions de bois de 70 à 140 t.ha-1 $^{-1}$. En RD Congo, Bisiaux et al., 2009 ont estimé à 120 tha $^{-1}$, la production de bois d'Acacia auriculiformis âgé de 12 ans. Toutefois, il convient de noter que les peuplements de la présente étude ont été plantés à forte densité $(2500$ pieds/ha).

Usages des produits des jachères arborées: Les quatre essences étudiées, en plus de présenter des aptitudes moyennes à bonnes pour le bois de chauffe, ont montré des qualités spécifiques pour la production de perches (Acacia mangium et Leucaena leucocephala) et pour la carbonisation (Acacia auriculiformis et Albizia lebbeck). La compatibilité des produits issus des jachères étudiées avec les usages du monde rural montre que ces espèces peuvent aider à résoudre, entre autres, l'épineux problème de la pénurie de bois énergie. En Côte d'Ivoire, des Légumineuses arborescentes (Acacia sp et Albizia sp.) plantées en association avec des cocotiers ont permis de satisfaire, partiellement, les besoins en bois de quelques villages de la zone littorale où l'on utilisait les palmes et les bourres de coco comme combustibles (Lekadou, 2009). En RD Congo, des paysans exploitant

\section{CONCLUSION}

Les quatre essences de jachères améliorées étudiées en zone forestière de Côte d'Ivoire ont montré des rythmes de croissance et des capacités de production de bois assez élevés, en seulement 6 ans. Le développement en hauteur a mis en relief les espèces à très forte croissance (Acacia mangium, Acacia auriculiformis et Leucaena leucocephala ). Quant à la production de bois, elle a mis en évidence les bonnes performances d'Acacia mangium et Acacia auriculiformis en termes d'accumulation de biomasse. Concernant les différents usages des produits issus des jachères arborées, chacune des essences étudiées s'est montrée plus apte à certaines utilisations qu'à d'autres. Ainsi, les tiges hautes et droites d'Acacia un gisement de 7000 ha d'Acacia auriculiformis produisent annuellement 8000 à 12.000 tonnes de charbon pour l'approvisionnement de la ville de Kinshasa (Bisiaux et al., 2009). Relativement à Acacia mangium qui a montré de faibles qualités de bois de chauffe et de carbonisation dans la présente étude, il y a lieu d'indiquer que les arbres étaient âgés de 6 ans et qu'en général, les rendements en charbon des essences peuvent s'améliorer avec l'âge des peuplements utilisés. La production de perches est une autre aptitude que certains peuplements de l'étude ont présentée. Selon Maldonado et Louppe (1999), les perches, piquets et poteaux issus des éclaircies du Teck peuvent se vendre respectivement à $600 \mathrm{~F}$ l'unité, $500 \mathrm{~F}$ le mètre et $5000 \mathrm{~F}$ l'unité, dans certaines métropoles ivoiriennes. Sans avoir la valeur économique du Teck certes, les mêmes produits issus des jachères améliorées de Légumineuses peuvent générer des revenus approximatifs. La carbonisation du bois des Légumineuses de notre étude en meules traditionnelles, a donné des rendements de 18 et $21 \%$ qui sont confirmés par Peltier et al. (2010) qui ont estimé entre 18 et $23 \%$ les rendements en charbon d'Acacia auriculiformis en RD Congo. Quant aux revenus de la vente du charbon de l'étude conduite à Oumé (1.720.000 F.ha-1 pour Acacia auriculiformis), ils paraissent plus élevés que ceux générés au Congo RD par les paysans du Projet Mampu (Peltier et al. (2010). Les gains nets de ces paysans sont de 2700 dollars US (1.350.000 F) pour 1,5 ha d'Acacia mangium abattus et carbonisés selon ces auteurs. En réalité, les gains estimés dans la présente étude sont élevés puisqu'ils ne font pas apparaitre les charges de production du charbon (frais d'abattage, de tronçonnage et de carbonisation) qui peuvent représenter jusqu'à $50 \%$ des recettes. Toutefois, ces revenus constituent pour le paysan, des ressources additionnelles non négligeables.

mangium et de Leucaena leucocephala ont montré de bonnes qualités de perches tandis qu'Acacia auriculiformis, Albizia lebbeck et Leucaena leucocephala ont affiché plus d'aptitudes de bois d'énergie (bois de chauffe et charbon), ce qui dénote de pouvoirs calorifiques appréciables. On peut retenir de cette étude que la plantation d'une jachère de Légumineuses arborescentes permet de produire, en peu de temps, une quantité non négligeable de bois d'utilisation courante et peut aider à freiner la déforestation des terroirs ruraux. Les ressources financières que le paysan peut tirer de la vente des produits des légumineuses arborescentes font de la plantation de ces nouveaux types de jachères, un 
moyen de diversification des revenus en milieu rural. Les plantations de légumineuses arborescentes peuvent donc participer à l'amélioration du bien être des populations rurales.

\section{REMERCIEMENTS}

Les auteurs remercient le CIRAD et l'IRD (France) pour le financement des activités de recherche et l'appui scientifique appréciable.

\section{REFERENCES}

Bernhard-Reversat F., Diangana D., Tsata M. 1993. Biomasse, minéralomasse et productivité en plantation d'Acacia mangium et Acacia auriculiformis au Congo. Bois et Forêt des Tropiques $n^{\circ} 238$ pp. 35-43.

Bisiaux F., Peltier R., Muliele J.C. 2009. Plantations industrielles et agroforesterie au service des populations des plateaux Batéké, Mampu, en République Démocratique du Congo. BFT.N ${ }^{\circ}$ 301(3) 21-32.

Dagnelie P. (1973) Théories et méthodes statistiques. Tome I et II. Presse Agronomique de Gembloux. Belgique.

De Rouw A. (1990). The invasion of Chromolaena odorata (L.) King and Robinson (ex. Eupatorium odoratum) and competition with the native flora in a rain forest zone, South West Côte d'Ivoire. J. of Biogeogr., 17 (1) $581.11 \mathrm{p}$.

Dommergues Y., Duhoux E. et H. G. Diem. 1999. Les arbres fixateurs d'azote : caractéristiques fondamentales et rôle dans l'aménagement des écosystèmes méditerranéens et tropicaux avec référence particulière aux zones subhumides et arides. Edition DUMAS, Saint-Etienne, 475 p.

Dupuy B. et N'guessan K. A. 1990. Utilisation des acacias pour régénérer les anciennes cocoteraies. Bois et Forêts des Tropiques, 230 : 15-29.

Dupuy B. et N'guessan K.A. 1991. Sylviculture d'Acacia mangium en Basse Côte d'Ivoire. Bois et Forêts des Tropiques, $N^{\circ} 225$, pp 24-32.

Floret Ch. et Pontanier R. 2000. La jachère en Afrique tropicale. Rôles, aménagements, alternatives. Actes du Séminaire International, Dakar, du 1316 avril 1999. Vol. 1, $777 \mathrm{p}$.

Gnahoua G.M, Oliver R., Nguessan K., Balle P. 2013. Production et retombées minérales des litières chez quatre espèces de légumineuses arborées, utilisées en amélioration de jachères, en zone forestière de Côte d'Ivoire. Journal of Applied Biosciences. Vol.72. pp 5800-5809.
Guillaumet J.L., Adjonohoun E. 1971. La végétation de la Côte d'Ivoire. Mémoires ORSTOM, ${ }^{\circ}$ 50, 157263.

Krisnawati, H., Kallio, M. and Kanninen, M. 2011 Acacia mangium Willd:: ecology, silviculture and productivity.CIFOR, Bogor, Indonesia. uww. cifor.cgiar.org

Lékadou T. T., N'guessan A., Konan J. L., Zakra N., YaoKouamé A., Allou K. 2009. Effet de la densité sur les paramètres dendrométriques d'Acacia auriculiformis en association avec le cocotier (Cocos nucifera L.) sur sables quaternaires en Côte d'Ivoire. Agronomie Africaine 21 (1) : 71 82

Maldonado G., Louppe D. 1999. Plantations villageoises de Teck. Bois et Forêts des Tropiques $N^{\circ} 262$ (4) $19-30$

Montagne P., Housseini M. 2000. Les jachères dans le bassin d'approvisionnement en bois de la ville de Niamey. Bois et Forêts des Tropiques, 2007, $\mathrm{N}^{\circ} 266$ (4) 55-59.

NAS (1984). Leucaena : Promising forage and tree crops for the Tropics. National Academy Press. Second edition. Washington D.C., 100 p.

Ouédraogo B. 2007. Filière bois d'énergie burkinabé : Structuration des prix et analyse de la répartition des bénéfices. Bois et Forêts des Tropiques, 2007, N² 294 (4) 75-88

Pardé J. et Bouchon P. 1988. Dendrométrie. Edition ENEF Nancy, $461 \mathrm{p}$.

Peltier R, Bisiaux F, Dubiez E, Marien J-N, Muliele J-C, Proces $P$ et Vermeulen C. 2010. De la culture itinérante sur brûlis aux jachères enrichies productrices de charbon de bois, en Rep. Dem. Congo

Prinsen J.H. 1986. Potential of Albizia lebbeck (Mimosaceae) as a tropical fodder tree. A review of literature. Queensland Department of Primary industries. Australia Tropical Grassland. Vol. 20, $n^{\circ}$ 2, 1986, pp 78-83.

Schröt G., Kolbe D., Pitty B., Zech W. 1995. Searching for criteria of the selection of efficient tree species for fallow improvment, with special 
Gnahoua et al. J. Appl. Biosci. 2014. Les jachères de légumineuses arborescentes: Sources potentielles de bois énergie et de service en Cote d'Ivoire

reference to carbon and nitrogen. Fertilizer Research, 42 : 297-314.

Sein, C.C., Mitlöhner, R. 2011 Acacia mangium Willd: ecology and silviculture. CIFOR, Bogor, Indonesia.

Singh A.N.,Raghubanshi A.S., Singh J.S. 2004. Comparative performance and restoration potential of two Albizia species planted on mine spoil in a dry tropical region, india. Ecological Engineering. 22: 123-140. 